\section{hommes}

\section{Hommes \& migrations}

Revue française de référence sur les dynamiques

migratoires

$1312 \mid 2015$

Diasporas iraniennes

\title{
Des couples iraniens en migration
}

Trajectoires d'intégration en Suède

\section{Yuko Suzuki}

\section{(2) OpenEdition}

1 Journals

\section{Édition électronique}

URL : http://journals.openedition.org/hommesmigrations/3496

DOI : 10.4000/hommesmigrations.3496

ISSN : 2262-3353

Éditeur

Musée national de l'histoire de l'immigration

\section{Édition imprimée}

Date de publication : 1 octobre 2015

Pagination : 71-79

ISBN : 978-2-919040-33-9

ISSN : 1142-852X

Référence électronique

Yuko Suzuki, «Des couples iraniens en migration », Hommes \& migrations [En ligne], 1312 | 2015, mis en ligne le 01 octobre 2018, consulté le 03 mai 2019. URL : http://journals.openedition.org/

hommesmigrations/3496; DOI : 10.4000/hommesmigrations.3496 


\section{DES COUPLES IRANIENS EN MIGRATION TRAJECTOIRES D'INTÉGRATION EN SUÈDE}

par YUKO SUZUKI, chercheur post-doctorante en anthropologie, UMR 7528 Mondes iranien et indien.

L'Iran représente le quatrième pays d'origine des migrants en Suède. L'engouement des Iraniens pour ce pays ne s'est pas démenti entre la première vague d'immigration faisant suite à l'avènement de la République islamique et la seconde après I'élection présidentielle de 2005. Si les réfugiés iraniens trouvent en Suède des conditions d'accueil favorables pour eux et leur famille, fondées sur la politique multiculturaliste, leur intégration culturelle dans cette nouvelle société censée n'avoir aucune similitude avec leur pays d'origine demande à chacun un énorme investissement.

La Suède est, après l'Allemagne, le deuxième pays européen d'accueil des immigrés iraniens ${ }^{1}$. Pour comprendre les raisons de cet engouement, plusieurs enquêtes qualitatives ont été menées auprès de réfugiés iraniens arrivés entre 1980 et 1988, puis entre 2007 et 2012 en Suède, menées début $2015^{2}$ dans les villes de Stockholm, Södertälje, Uppsala et Göteborg, et complétées par des statistiques. Ces recherches ont pour objectif de mesurer l'influence de la politique des pays de départ et d'accueil sur la vie des immigrés et de mieux cerner les effets des moyens de communication numérique sur les liens entre les personnes. Pour obtenir des informations précises lors d'une mission assez courte, j’ai bénéficié de deux réseaux iraniens : le réseau tribal de la province iranienne de Kohgiluye-va-Boirahmad, qui est le lieu de mes recherches depuis plus de vingt ans, et celui d'un réfugié à Paris, ancien membre de l'Union des organisations d'étudiants en Iran, appelée "Bureau pour le renforcement de l'unité", daftar-e tahkim-e vahdat en persan. Ces deux réseaux mobilisés dans trois pays (France, Suède et Iran) m'ont permis de mettre en place une vingtaine d'entretiens et des séjours dans quatre familles iraniennes, dès mon premier voyage en Suède. 
L'immigration

iranienne en Suède : conjonction de deux contextes politiques

16,5 \% de la population de la Suède sont nées à l'étranger selon les statistiques de $2014^{3}$. Dans ce pays nordique dont la densité démographique est très faible, l'immigration est indispensable: sans immigration, la croissance démographique serait négative ${ }^{4}$. L'immigration constitue notamment une réponse immédiate pour maintenir le taux de jeunes dans ce pays vieillissant ${ }^{5}$. Fin 2014, il y avait 68436 résidents nés en Iran (graphique 1), dont 35694 hommes et 32742 femmes. Ce chiffre regroupe principalement des étudiants, des réfugiés et des demandeurs d'asile. L'Iran représente le quatrième pays d'origine des étrangers en Suède, après la Finlande (158 488), l'Irak (130 178) et la Pologne (81 697).

En plus de la première génération d'Iraniens installés dans le pays, près de 32000 citoyens suédois ont au moins l'un de leurs

En 1976, il n'y avait que 1412 immigrés iraniens en Suède, dont la majorité étaient étudiants. Après la révolution, les entrées annuelles n'ont pas dépassé 500 (579 en 1980), jusqu'en 1984 où elles doublent avec 1074 personnes. parents né en Iran. Grâce à la politique suédoise de maintien de la connaissance de la langue maternelle, la deuxième génération parle couramment le persan. De ce fait, nous estimons à plus de 100000 les persanophones de Suède. Dans ces conditions, il métait souvent nécessaire de chuchoter avec mes informateurs pour ne pas nous faire entendre des persanophones, très nombreux dans les grandes villes.

En 1976, il n'y avait que 1412 immigrés iraniens en Suède, dont la majorité étaient étudiants ${ }^{6}$. Après la

\section{/// Graphique 1 : Évolution du nombre de personnes nées en Iran résidant en Suède.}

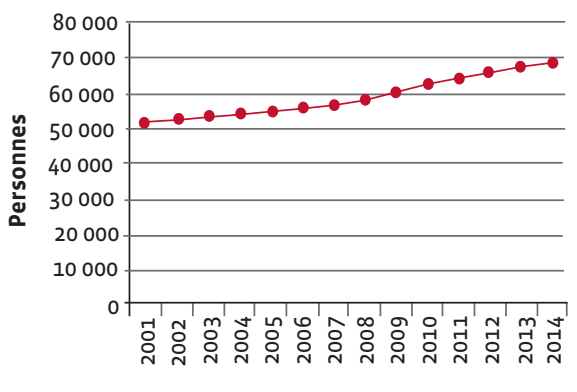

Année

Source : Statistiska centralbyrån (SCB), Centre statistique suédois, www.scb.se.

révolution, les entrées annuelles n'ont pas dépassé 500 (579 en 1980), jusqu'en 1984 où elles doublent avec 1074 personnes. Le chiffre augmente régulièrement jusqu'en 1988, où 6203 Iraniens sont enregistrés par les autorités. Depuis l'année 1984, cette augmentation est le résultat de l'exil des membres des partis libéraux et communistes, à la suite des conflits post-révolutionnaires qui ont opposé les partisans de la révolution à partir de 1981. Par ailleurs, la Suède est peu à peu reconnue comme une destination de choix par les opposants du régime islamique. Cependant, depuis la prise d'une série de mesures pour un contrôle plus strict de l'immigration par le gouvernement suédois en 1989, le nombre des immigrés iraniens a baissé de 5398 en 1989 à 2854 en 1992. La deuxième vague d'immigration iranienne en Suède a commencé en 2006 avec environ 2000 entrées annuelles pour culminer en 2010 avec 2820 immigrés (graphique 2).

Le pic de ces courbes est lié au double contexte politique iranien et suédois. Après l'élection 
d'Ahmadinejad à la présidence en 2005, un bon nombre de partisans du président sortant, Khatami, déçus et menacés, sont partis du pays, alors que la gratuité de la scolarité et la pratique de la langue anglaise (au niveau du master dans les universités suédoises), ainsi que le modèle politique social-démocrate attiraient de nombreux étudiants iraniens vers la Suède.

Ensuite, la réélection contestée dudit président en 2009 et la condamnation d'un grand nombre de ceux qui avaient contesté les résultats ont conduit ces derniers à l'exil. Or, depuis 2011, la fin de la gratuité des frais d'études universitaires en Suède a découragé une partie des étudiants iraniens qui voulaient s'y installer.

Même si on estime que le niveau de scolarisation des immigrés iraniens en Suède est élevé, trouver un emploi à l'étranger reste difficile pour eux. Selon les statistiques nationales en 2006, seulement $54,4 \%$ de la population née en Iran et âgée de 20 à 64 ans sont considérés comme salariés (57,7 pour les hommes contre 50,6 pour les femmes), soit bien moins que le reste de la population, même née à l'étranger. Comme le montre le graphique 3 , une grande majorité des Iraniens ont des revenus moyens ou bas, leur salaire n'atteint pas le revenu annuel moyen, environ 300000 couronnes suédoises (SEK) en 2006.

\section{Le parcours de l'accès aux droits des réfugiés}

Monsieur A., 31 ans, issu d'une classe sociale aisée, est venu en Suède en 2010 et vit avec sa femme iranienne qu'il a épousée en 2008. Il a répondu à mes questions sans grande hésitation ni méfiance, tout en remarquant qu'en Suède, il n'avait jamais parlé de ses affaires familiales ni de son passé même avec ses amis iraniens.
Inscrit depuis 2002 dans une université renommée de Téhéran, il était membre du conseil principal de l'organisation des étudiants - qui appartenait à la fraction moderne $e^{7}$ du Bureau pour le renforcement de l'unité - pendant la présidence de Khatami. Après lélection présidentielle de 2005,

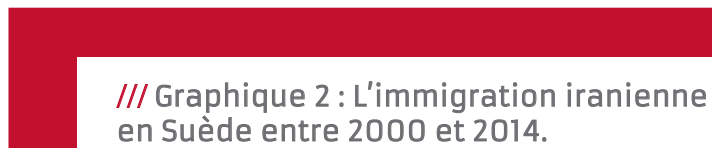
en Suède entre 2000 et 2014.

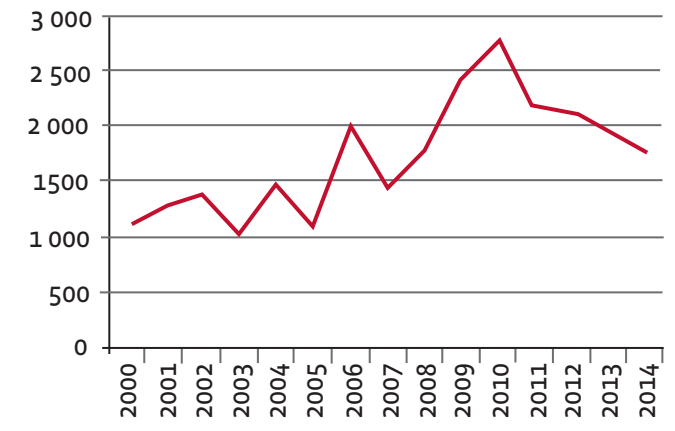

Source : Statistiska centralbyrån (SCB), Centre statistique suédois, http://www.scb.se.

les activités de cette fraction furent considérablement restreintes sous la présidence d'Ahmadinedjad. En 2007, le bureau est officiellement fermé et monsieur $\mathrm{A}$. arrêté et condamné à deux ans de prison ferme, plus cinq ans de sursis. Travaillant pour un journal en ligne, il a continué de vivre dans l'actualité politique de l'Iran. Après l'élection présidentielle de 2009, il fut actif dans le Mouvement vert, manifestations spontanées qui se sont soldées par des confrontations dramatiques entre manifestants et forces de l'ordre.

Convoqué au tribunal et effrayé par la perspective de la prison, il s'exile à la fin du mois de mai 2010 et part pour la Suède grâce au soutien d'un ami iranien déjà installé dans ce pays. Ce dernier, ayant 
/// Graphique 3 : Revenus des salariés selon le pays de naissance.

\section{Salariés nés en Iran}

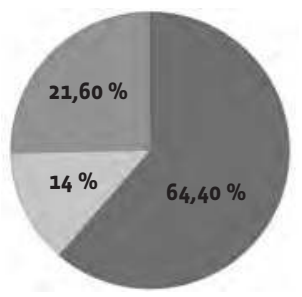

Salariés nés à l'étranger

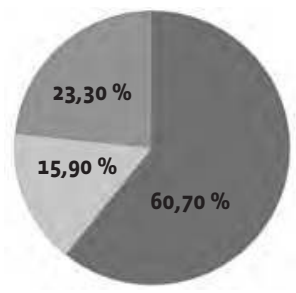

Salariés nés en Suède

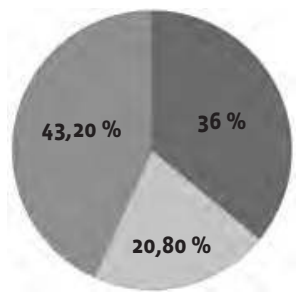

0 à 155 mille SEK (o à $16490 €$ )

155,1 à 241,4 mille SEK (16490à 25681€)

Supérieur à 241,4 mille SEK (supérieur à $25692 €$ )

Source : "Average monthly salary", in Statistiska centralbyrån $(\mathrm{SCB})$, Centre statistique suédois, www.scb.se.

une certaine influence au sein du ministère des Affaires étrangères de Suède, lui a préparé son visa touristique, alors que son père, haut fonctionnaire, multiplie les efforts pour qu'il ne soit pas privé du droit de sortie du pays. Grâce à cette collaboration, il a pu partir d'Iran par avion, de manière légale.

En Suède, à l'expiration de son visa touristique, il demande l'asile au Bureau de l'immigration. Ayant obtenu le statut de réfugié, il vit de son épargne et de la vente d'accessoires et de pièces d'or reçus en cadeau à l'occasion de son mariage, tout en louant une chambre au nord de Stockholm qui coûte 4500 SEK (alors $440 €$ ). En novembre 2010, à peine trois mois après, il obtient la carte de résident en tant que réfugié politique. Il bénéficie ensuite d'une allocation mensuelle de 6000 SEK (environ $600 €$ ), à condition de suivre le programme de langue suédoise géré par la commune.

Sa femme, madame B., journaliste de presse iranienne, le rejoint en Suède en avril 2011. Dès son arrivée, en tant quépouse d'un résident, elle bénéficie de la protection sociale suédoise. Après cinq mois seulement, le couple achève le cursus de langue suédoise qui dure en général deux ans. Par la suite, tous deux s'inscrivirent en master à l'université.

\section{Reprise d'études et mise à distance des activités politiques}

En tant qu'étudiants, ils reçoivent chacun une bourse et un prêt, pour un montant de 9000 SEK (1000 €) par mois. Ce couple gagne désormais 18000 SEK, soit plus de $2000 €$, même si plus de $60 \%$ de cette somme devront être remboursés après leurs études. Monsieur A. avoue qu'il n'a jamais dû demander d'argent à sa propre famille ni à la famille de sa femme depuis son arrivée en Suède. Après son master, monsieur $\mathrm{A}$. reprend un master en économie politique, alors que madame B. choisit la licence de l'École supérieure des sciences sociales. Il se prépare au doctorat, tandis 
que sa femme entend suivre une formation d'assistante sociale.

Je l'ai interrogé sur les changements de sa vision politique et de son mode de vie après son arrivée en Suède: "Je n'ai plus d'activités politiques depuis mon arrivée en Suède. Je n'ai fait que mes études depuis quatre ans (...). Les problèmes sociaux, culturels et politiques en Iran m'intéressent encore comme par le passé. Mais je les observe maintenant en tant que scientifique. Il y a des personnes qui font des manifestations et des meetings politiques tous les jours à Stockholm. Mais la plupart de ces gens n'ont pas réussi dans la société suédoise : ils sont toujours dépendants des allocations familiales sans avoir de métier. Quand on est engagé dans la politique iranienne, on n'a pas de temps pour maîtriser la langue suédoise et faire des études universitaires. Je ne veux pas me trouver dans la même situation, vingt ans après." Comme exemple d'Iraniens ayant réussi dans ce pays, il cite Mehrdad Darvishpour, sociologue de l'université de Mälardalen et Ardala Shekarabi, à peine âgé de 35 ans, nommé ministre des Affaires civiles en octobre 2014 par le Premier ministre Stefan Löefven du Parti social-démocrate suédois des travailleurs.

\section{Des stratégies d'autonomisation}

Monsieur C., né en 1970 dans un village de la province de Kohgiluye-va-Boirahmad, vivait dans une région tribale jusqu'à son entrée à l'université. Son frère, militant d'un parti communiste pendant et après la révolution islamique, chassé par le régime iranien actuel, s'est exilé en Suède en 1983. En 2007, monsieur C. lui fait sa première visite. En 2010, l'ambassade de Suède en Iran délivre à monsieur $C$. ainsi qu'à sa femme et à leurs trois enfants les visas de tourisme qu'ils demandaient pour aller soigner un frère handicapé. Après les trois mois en Suède, son fils, satisfait de l'école suédoise, a exprimé le désir d'y vivre, alors que monsieur $C$. souhaitait retourner en Iran. Il s'est servi de son histoire personnelle pour demander l'asile en Suède : considéré comme activiste dans l'opposition au régime actuel par les autorités iraniennes, il avait fait trois mois de prison après l'élection présidentielle de 2009. Sa demande a été acceptée un an et trois mois après le dépôt de dossier. Durant ce délai, avec une seule subvention limitée aux frais alimentaires, il a été conduit à épuiser son épargne et à chercher des emplois informels. Or, pour un homme tribal titulaire d'un diplôme universitaire, viril et fier, demander à un inconnu un "petit" travail était humiliant. Monsieur C. a donc inventé sa propre méthode de recherche d'emploi : il a envoyé par SMS son profil et sa recherche de travail à toutes les personnes inscrites dans le livret des entrepreneurs iraniens en Suède. Il a ainsi reçu plusieurs offres de travaux subalternes.

Dès l'acquisition du statut de réfugié avec la carte de résident permanent, monsieur $C$. bénéficie de la protection sociale suédoise comme les Suédois. Son existence est désormais assurée : le Swedish Migration Board lui propose un appartement de $110 \mathrm{~m}^{2}$ dans une commune du sud du comté de Stockholm. La totalité du loyer est subventionnée, la majorité de l'allocation de logement étant payée au titre des trois enfants. De plus, la part des allocations familiales pour le troisième enfant est plus élevée que celle des deux premiers (1500 SEK, contre

"Quand on est engagé dans la politique iranienne, on n'a pas de temps pour maitriser la langue suédoise et faire des études universitaires. Je ne veux pas me trouver dans la même situation, vingt ans après." 1200 SEK). Enfin, la société suédoise investit beaucoup sur les enfants : les frais scolaires sont presque gratuits jusqu'à lâge de 18 ou 20 ans selon la commune; les jeunes n'ont pas à payer les consultations médicales; le prêt d'argent et la bourse sont donnés à tous les étudiants à temps complet. Avec ce système, les enfants peuvent devenir financièrement indépendants entre 17 et 18 ans. 


\section{Les atouts du multilinguisme scolaire}

Après six mois de travail comme jardinier, monsieur C. recommence ses études de langues suédoise et anglaise, et de mathématiques pour préparer la formation d'auxiliaire médical. Avec son diplôme iranien de bac +4 en histoire, il ne trouvait que du travail manuel. Il vise ainsi le marché du travail paramédical, qui est l'un des domaines les plus riches en emplois

En Suède, les enfants des immigrés sont encouragés à

apprendre non seulement le suédois mais également leur

langue maternelle à partir de l'école maternelle jusqu'à

la fin du lycée. qu'est la Suède.

"Il est très difficile d'entrer dans la société suédoise. Elle est presque close envers les étrangers qui restent toujours en marge sans avoir aucune dans ce pays vieillissant relation amicale avec les Suédois. L'université est la porte pour entrer dans cette société. Mes enfants passent souvent la nuit chez leurs amis suédois et l'inverse est tout aussi vrai. Mais, quand leurs parents viennent les chercher chez moi, ils ne me font qu'une salutation de pure convenance. Une relation plus intime ne commence pas par ce contact." Il sait que son problème ne vient pas de la discrimination raciale mais plutôt de la barrière de la langue. Ses enfants se sont très bien intégrés, au point qu'ils ne se distinguent plus de leurs amis suédois aux cheveux blonds et aux yeux bleus. Ils sont devenus bilingues et pratiquent le suédois entre eux et le persan avec leurs parents. En Suède, les enfants des immigrés sont encouragés à apprendre non seulement le suédois mais également leur langue maternelle à partir de l'école maternelle jusqu’à la fin du lycée. Un ou deux cours de langue d'origine sont programmés par semaine dans le cadre du projet du ministère de l'Éducation.
De plus, l'école conseille aux parents et aux enfants de pratiquer cette langue à la maison.

La promotion du multilinguisme ${ }^{8}$ s'appuie sur une politique multiculturaliste : l'intégration des immigrés ne peut être réussie que si le nouveau venu se sent comme chez lui. Dans ce cadre, le projet d'enseignement des langues d'origine est mis en œuvre depuis 1977 par l'Éducation nationale ${ }^{9}$, afin de mieux transmettre les cultures des immigrés à leurs enfants ${ }^{10}$.

Monsieur C. apprécie beaucoup cette politique qui permettra un jour à ses enfants d'éviter de passer par une crise d'identité.

\section{Le poids des représentations tribales et religieuses}

Sa seule gêne demeure la liberté sexuelle et l'éducation à la sexualité. Il est choqué par le fait que son fils de treize ans ait dans la poche un préservatif distribué à l'école. Il n'est pas acceptable pour lui que l'école instruise les enfants sur la contraception sans leur interdire les rapports sexuels. Monsieur $C$. vient d'une société tribale où le rapport sexuel avant le mariage est encore tabou. L'offense à la pudeur virginale d'une fille est une atteinte à la dignité de toute sa famille. Il y a une trentaine d'années, les hommes dans la société tribale d'Iran jouaient même leur vie pour la défendre. Tout en étant en Suède, il vit encore dans la société tribale grâce aux communications téléphoniques ou à l'Internet. Les valeurs sociales et les rivalités entre familles lui imposent d'honorer sa famille en cachant ses métiers effectués en Suède pour laisser penser qu'il continue ses études universitaires. Même dans sa région natale, longtemps isolée des tendances des grandes villes, l'obtention d'un master ou d'un doctorat (en particulier d'une université étrangère) est devenue ces

8. La Suède a six langues officielles, même si le suédois est reconnu en tant que langue majeure du pays depuis décembre 2005 par décision du Parlement. De surcroît, environ deux cents langues de migrants, non officielles, sont pratiquées dans ce pays. Voir Inger Lindberg, "Multilingual education: a Swedish perspective”, in Marie Carlson, Annika Rabon, Fatma Gök, "Education in 'Multicultural' Societies - Turkish and Swedish Perspectives”, Swedish Research Institute in Istanbul, Transactions, vol. 18, 2007, pp. 71-90. 9. Inger Lindberg, op. cit., p. 76. 10. Stéphane Pringuet, "Assimilation danoise, ou multiculturalisme suédois; analyse de deux modèles d'intégration", in Opinion internationale, 2011. 
dernières années un trophée social. Madame D., son épouse née en 1977, portait un foulard islamique. Fiancée à lâge de 17 ans, elle s'est mariée après son bac avec le fils du frère de sa grandmère maternelle. Sa carte de résident permanent en Suède lui a été délivrée deux ans après son arrivée. Elle touche, depuis, son propre salaire versé par le Bureau de l'emploi en suivant les cours de langue suédoise. Actuellement, elle va à l'école tous les après-midi pour deux heures de cours de suédois et d'anglais, sauf les week-ends. Après six mois, elle devra choisir entre trouver un emploi ou obtenir un prêt afin de poursuivre des études supérieures. "J'envisage de demander un prêt étudiant. Le métier d’auxiliaire médical m’intéresse. (...) Et puis, si je fais des progrès en suédois, je voudrais suivre la formation d'infirmière. En Iran, j'étais femme au foyer. Mais maintenant j'aimerais avoir un métier ici, car c'est normal que les femmes travaillent en Suède."

Depuis l'obtention de sa carte de résident, elle bénéficie aussi d'un accompagnement personnalisé de la part d'une assistante sociale qui la renseigne sur la possibilité d'une vie autonome pour une femme dans la société suédoise. Madame D. n'est plus en contact avec les autres immigrées iraniennes, bien que dans un premier temps après son arrivée, elle ait participé aux "agapes des vœux" (sofre-ye nazr) à Stockholm. Une des raisons de cet éloignement est le port du foulard islamique. Celui-ci est admis en Suède comme les autres voiles islamiques (sauf la burqa) dans les espaces publics. Mais, selon elle, la plupart des Iraniennes immigrantes n'apprécient pas les personnes voilées. "Je le considère comme une habitude depuis mon enfance, plutôt que comme un signe de religiosité. Sans foulard, je suis gênée, comme les Suédoises sont embarrassées avec un foulard." Elle pense que c'est son choix personnel et qu'elle n'a pas le droit d'intervenir dans le choix de ses deux filles.

\section{L'exil au service d'une promotion sociale}

Très souriant, monsieur G., âgé de 32 ans, portant jean et capuche comme un étudiant suédois, semble chercher le style vestimentaire simple et raffiné des universitaires suédois. Turkmène par son père, appartenant à l'islam sunnite dans le pays dont la religion nationale est l'islam chiite, il possède sans doute une certaine habilité à communiquer et à vivre dans une autre culture en tant

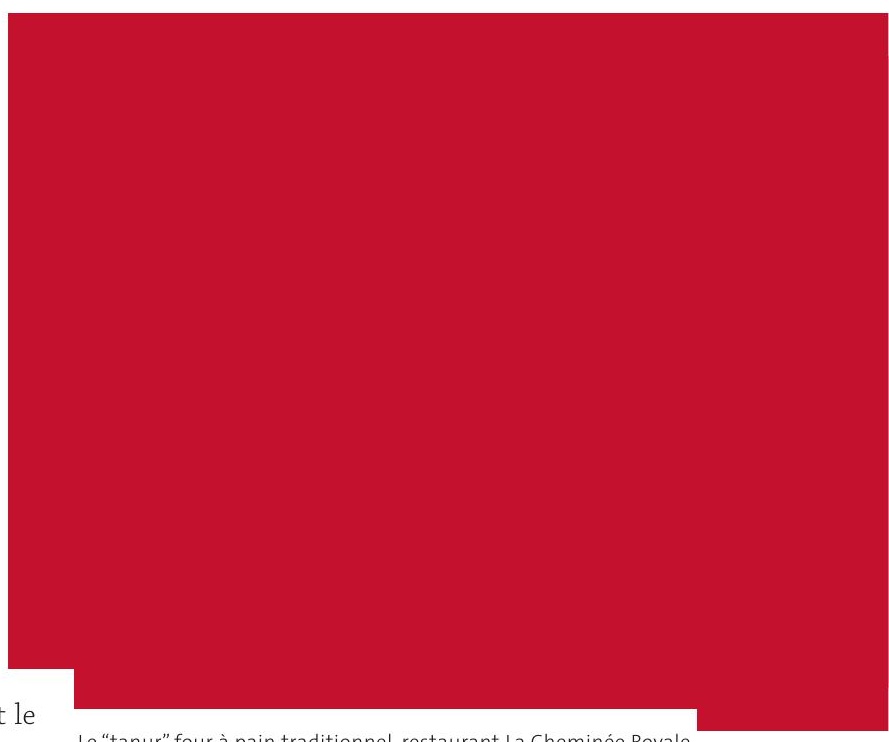

Le "tanur" four à pain traditionnel, restaurant La Cheminée Royale, Paris, 1991. @ IsABELle EshraghI.

que membre d'une minorité ethnique et religieuse. Il a été un pratiquant sérieux durant son adolescence, où il étudiait l'islam sunnite à l'école religieuse de sa province, le Golestân. Depuis 2002, il était membre du Bureau pour le renforcement de l'unité à l'université de Gilan. À la fin de ses études de chimie, à lâge de 24 ans, il est venu en Suède pour étudier la biotechnologie en master à l'univer- 
sité de Borås, située dans le comté de Västra Götaland. Après l'obtention de son premier master, il a travaillé pendant un an dans un laboratoire de l'université d'Uppsala. Ensuite, il a obtenu en 2011 son deuxième master de nanotechnologie de l'université de Göteborg. Du point de vue financier, il est plutôt favorisé par son milieu familial, trois frères aînés étant devenus avocats. Avec ses revenus de travaux saisonniers et l'aide de sa famille, il a mené sa vie d'étudiant sans subventions gouvernementales. Il a demandé l'asile en qualité de réfugié politique pour rester en Suède et a reçu sa carte de résident un mois et demi après. L'autorité compétente a tenu compte de ses activités politiques telles que la rédaction d'articles dans des journaux iraniens et sa participation au débat Les Iraniens arrivés politique dans une émission après 2006 en Suède sont de radio de Suède.

davantage issus des classes moyennes et sont capables de financer le voyage et le début du séjour. De plus, ils sont mieux informés sur la société suédoise que ceux arrivés dans les années 1980.

À la fin de ses études, il a fait divers petits boulots pour gagner sa vie, comme serveur dans un café ou un restaurant pendant un an, jusqu'à sa prise d'emploi dans un institut de recherche. Depuis novembre 2012, il occupe un poste de chercheur en nanopolymère et s'est récemment inscrit pour un doctorat de nanotechnologie. Il gagne déjà 32 à 35000 SEK, environ 3700 euros, par mois. Après le paiement du loyer qui coûte 1100 euros, il lui reste encore assez d'argent pour mener une vie aisée avec sa famille. Dans quatre ans, avec son doctorat, son salaire grimpera encore de $25 \%$. La valorisation des études comme moyen de promotion sociale en Iran a été efficace pour lui. "Dans ma région, Gorgân [région des Turkmènes au nord de l'Iran], il n'y a que les études pour gagner la vie, sinon on devient paysan." Outre ses trois frères avocats, il a un frère cadet vétérinaire et ses deux cadets sont étudiants en droit, tandis que son père a été longtemps paysan.

Le changement du climat de la politique iranienne depuis l'avènement d'Ahmadinejad à la présidence l'a conduit à quitter l'Iran. En 2005, juste après l'élection présidentielle, ce proche du parti réformiste a été banni du Bureau pour le renforcement de l'unité de l'université par la fraction minoritaire en faveur du régime iranien.

Malgré son désir d'aller en Angleterre, il a choisi la Suède pour la gratuité de la scolarité. Il est venu en compagnie de 9 camarades de son université. Selon ses dires, en 2007, 280 étudiants iraniens ont été admis dans des universités suédoises. Sa classe de biotechnologie comptait 5 Iraniens sur 20 étudiants, alors que la classe de management comportait 18 Iraniens sur 20. Actuellement, monsieur G. vit dans un appartement moderne et relativement luxueux de $100 \mathrm{~m}^{2}$ avec sa famille recomposée : sa femme et deux enfants, dont l'aîné est né du premier mariage de celle-ci.

\section{Sortir de l'état de minorité}

Son épouse, madame H., était sa petite amie, étudiante comme lui à l'université de Gilân. Sa famille n'était pas satisfaite de l'appartenance religieuse de son ami, l'islam sunnite. En outre, l'oncle maternel de madame H., proche du Guide suprême de l'État iranien, n'appréciait pas ses activités politiques de réformiste et partisan de Khatami. Ainsi, monsieur G. a failli se faire exiler de son pays sous la menace de la famille de madame H., celle-ci étant obligée d'épouser un homme choisi par ses proches.

Plusieurs années après leur séparation, en 2011, les anciens amoureux se revoient au Danemark et, dix jours après leurs retrouvailles, monsieur $G$. fait sa demande en mariage. En octobre 2012, madame H. se rend à Göteborg pour se marier. Selon la loi iranienne, dans le cas d'une femme divorcée, la permission des parents n'est pas requise pour un nouveau mariage. Le couple organise un mariage à la méthode suédoise, sans rien dire à la famille de l'épouse. Pour autant, se sentant coupable de l'échec du premier mariage, la famille de madame H. ne s'oppose plus à ce deuxième. 
Rebutée par les démarches administratives pour obtenir sa carte de résident et comblée par la naissance de sa fille, madame $H$. n'a pas eu le temps d'apprendre la langue suédoise avant février 2015. Elle communique donc en anglais avec les Suédois. Depuis l'inscription de sa fille de 15 mois à la crèche, elle s'est inscrite en cours de suédois afin de trouver un emploi.

Pour ce couple, quitter l'Iran pour arriver en Suède a été la clé de son existence actuelle : libéré du statut de la minorité religieuse et ethnique en Iran, monsieur G. est devenu un simple étranger en Suède, tandis que madame H., émancipée de l'emprise de sa famille, se prépare à devenir une femme autonome.

Les relations de monsieur G. avec les Iraniens en Suède sont limitées aux amis des années d'études. Il a bien eu des discussions avec d'anciens militants de partis de gauche iraniens, mais depuis qu'il s'est tourné vers l'islam en Suède - selon ses dires, il est devenu plus religieux que jamais -, il n'arrive plus à trouver de point d'accord avec eux. Il conserve ainsi une certaine distance avec les ressortissants iraniens des années 1980. Vis-à-vis des Suédois, il est incontestablement l'Iranien qui s'est le mieux inséré dans la société suédoise parmi mes informateurs. Il avait même une petite amie suédoise à une époque et, actuellement, il reçoit chez lui des amis suédois. Il avoue pourtant qu'il reste difficile de se faire des amis parmi les Suédois, même pour lui qui est particulièrement sociable.

\section{Conclusion}

Les Iraniens arrivés après 2006 en Suède sont davantage issus des classes moyennes et sont capables de financer le voyage et le début du séjour. De plus, ils sont mieux informés sur la société suédoise que ceux arrivés dans les années 1980. Ils ont choisi la Suède d'eux-mêmes. Grâce à Internet,
Skype, Viber ou Facebook, le lien avec leur famille et leurs amis n'a jamais été rompu. Ils n'ont pas connu la solitude ni la nostalgie de l'Iran, comme les éprouvaient les anciens migrants. Ils n'ont jamais eu le complexe de se trouver au second rang, comparé aux Iraniens résidant en France ou en Angleterre qui ont joué un grand rôle sur la scène politique pendant et après la révolution islamique.

Leurs rapports avec les autorités iraniennes sont également différents. Si certains craignent une peine d'un ou deux ans d'emprisonnement à leur retour en Iran, aucun ne ressent cela comme une menace. Sur ce point, ils font même confiance aux autorités iraniennes, au contraire des anciens communistes ou royalistes. Ils n'ont ni haine ni attachement tenace envers le régime iranien actuel. Les immigrés récents profitent pleinement des services sociaux suédois et d'une politique basée sur le multiculturalisme, l'égalitarisme et l'individualisme : l'État assure le bien-être de chaque être humain (même non-citoyen) comme cela est déclaré dans la Constitution suédoise ${ }^{11}$. Pourtant, malgré cette assurance matérielle et culturelle, ils continuent en grande partie à se sentir marginalisés dans la société suédoise à cause du manque de communication avec les autochtones ${ }^{12}$. 